numerical method based on the theory of Kryloff and Bogoliuboff will now be apparent.

University of Ife

Ibadan, Nigeria p. 471 .

1. N. M. Kryloff \& N. Bogoliuboff, Izv. Akad. Nauk S.S.S.R. Ser. Mat. Fiz., 1929,

2. J. C. Burkill, The Theory of Ordinary Differential Equations, University Mathematics Texts, Oliver and Boyd, Edinburgh, 1956.

3. N. W. McLachlan, Theory and Application of Mathieu Functions, Clarendon Press; Oxford, 1947. MR 9, 31.

\title{
Integration Rules of Hypercubic Symmetry over a Certain Spherically Symmetric Space
}

\section{By J. N. Lyness}

Abstract. A theory of integration rules suitable for integration over a hypercube and having hypercubic symmetry has recently been published. In this paper it is found that, with minor modification, this theory may be directly applied to obtain integration rules of hypercubic symmetry suitable for integration over a complete $n$-dimensional space with the weight function $\exp \left(-x_{1}{ }^{2}-x_{2}{ }^{2} \cdots-x_{n}{ }^{2}\right)$. As in the case of integration over hypercubes, an $n$-dimensional rule of degree $2 t+1$ may be constructed requiring a number of function evaluations of order $2^{t} n^{t} / t$ !, only.

1. Introduction. In this paper we are interested in generalising the theory and results of investigations about the use of symmetric integration rules for a hypercube, given in Lyness [2] and [3] which we refer to as Part I and Part II, respectively. The particular generalisation that we consider here is the construction of rules of the type

$$
\begin{aligned}
\int_{-\infty}^{\infty} \int_{-\infty}^{\infty} \cdots \int_{-\infty}^{\infty} \exp \left[-x_{1}{ }^{2}-x_{2}{ }^{2} \cdots-x_{n}{ }^{2}\right] f\left(x_{1}, x_{2}, \cdots, x_{n}\right) d x_{1} d x_{2} \cdots d x_{n} \\
\simeq \sum A_{i} f\left(\iota_{1 i}, \mathfrak{s}_{2 i}, \cdots, \text { s } n i\right) .
\end{aligned}
$$

Such integration rules have been considered before (Stroud and Secrest [4]). It is conventional to term such a rule to be a rule of degree $d$ if the approximate equality may be replaced by an exact equality whenever $f$ is a multinomial of degree less than or equal to $d$.

Most of the results about synmetric rules for integration over hypercubes in Parts I and II may be derived in almost identical form for this integral. To avoid unnecessary repetition, we refer to Parts I and II for the details of the derivations of such results; we indicate here only the differences or modifications in these results as they occur.

Received October 12, 1964. This research was supported in part by USAF Grant No. 62-400 to the University of New South Wales. 
2. The Integration Operator, $I^{(r)}$. We define the symmetric integration rule operators $R$ and $R$ exactly as in Section 1 of Part I with the number $a$ set, for convenience, equal to $\frac{1}{2}$. In this case, all the operator algebra connected with rules, such as the projection of and the extension of rules and the number of points required by a rule, as described in Sections 2 and 3 of Part II, and in Sections 1 and 5 of Part I, may be used quite independently of the region of integration.

Without a definition of an integration operator and its connection with the rule operators, the rule formalism is of little practical value. We introduce instead of $(I ; 1.1)$ and $(I ; 1.7)$ an $r$-dimensional integration operator $I^{(r)}$ defined by

$$
I^{(n)} f=\frac{\int_{-\infty}^{\infty} \int \cdots \int \exp \left[-x_{1}^{2}-x_{2}^{2} \cdots-x_{n}{ }^{2}\right] f d x_{1} d x_{2} \cdots d x_{n}}{\int_{-\infty}^{\infty} \int \cdots \int \exp \left[-x_{1}{ }^{2}-x_{2}{ }^{2} \cdots-x_{n}{ }^{2}\right] d x_{1} d x_{2} \cdots d x_{n}} .
$$

The denominator is equal to $(\pi)^{n / 2}$.

We turn our attention to finding rules $R^{(r)}$ which are useful approximations to $I^{(r)}$ in the sense that

$$
R^{(r)} f \simeq I^{(r)} f
$$

the approximation being exact at least when $f$ is a constant function. For example, a possible two-dimensional rule is

$$
R^{(2)}=R(\sqrt{ } 2,0)
$$

and using the definition in Part I, we may write

$$
R^{(2)} f=\frac{1}{4}\left(f\left(\frac{1}{\sqrt{ } 2}, 0\right)+f\left(-\frac{1}{\sqrt{ } 2}, 0\right)+f\left(0, \frac{1}{\sqrt{ } 2}\right)+f\left(0,-\frac{1}{\sqrt{ } 2}\right)\right) .
$$

The corresponding integral given by $(2.1)$ is

$$
I^{(2)} f=\frac{1}{\pi} \int_{-\infty}^{\infty} \int \exp \left[-x^{2}-y^{2}\right] f(x, y) d x d y
$$

and so, in the form of $(1.1)$, the integration rule $R(\sqrt{ } 2,0)$ leads to the approximate equality

$$
\begin{aligned}
& \int_{-\infty}^{\infty} \int \exp {\left[-x^{2}-y^{2}\right] f(x, y) d x d y } \\
& \simeq \frac{\pi}{4}\left[f\left(\frac{1}{\sqrt{ } 2}, 0\right)+f\left(-\frac{1}{\sqrt{ } 2}, 0\right)+f\left(0, \frac{1}{\sqrt{ } 2}\right)+f\left(0,-\frac{1}{\sqrt{ } 2}\right)\right] .
\end{aligned}
$$

This differs from the corresponding form for integration over a hypercube because of the "normalising" factor on the right-hand side.

3. The Error Coefficients. The error expansion described in Sections 2 and 3 of Part I may be carried out in the same way. In one dimension we find, by successive integration by parts, that an expansion of the type

$$
\Re(\alpha) f-I^{(1)} f=d_{2}(\alpha) I^{(1)} f^{(2)}+d_{4}(\alpha) I^{(1)} f^{(4)}+\cdots
$$


exists, where $f^{(r)}$ is the $r$ th derivative function of $f$. The coefficients $d_{2 r}(\alpha)$ are termed error coefficients. We may obtain a generating function for the error coefficients by inserting into (3.1) the function

$$
f=2 \cosh \phi x .
$$

Carrying out the integrations analytically and setting

$$
d_{0}(\alpha)=1,
$$

we find, after some rearrangement,

$$
\sum_{r=0}^{\infty} d_{2 r}(\alpha) \phi^{2 r}=\cosh \frac{\phi \alpha}{2} \exp \left(-\frac{\phi^{2}}{4}\right) \equiv G(\alpha, \phi) .
$$

This generating function may be used to find expressions for the error coefficients, namely,

$$
d_{2 r}(0)=(-1)^{r} /\left(2^{2 r} r !\right)
$$

and

$$
d_{2 r}(\alpha)=2^{-2 r} \sum_{s=0}^{r} \frac{(-1)^{r-s} \alpha^{2 s}}{(2 s) !(r-s) !} .
$$

A convenient expression for calculating the error coefficients is

$$
d_{2 s}(\alpha)=\frac{d_{2 s}(0)}{2^{2 s}}+\frac{\alpha^{2}}{2 !} \frac{d_{2 s-2}(0)}{2^{2 s-2}}+\cdots \frac{\alpha^{2 s-2}}{(2 s-2) !} \frac{d_{2}(0)}{2 !}+\frac{\alpha^{2 s}}{2 s !} d_{0}(0),
$$

in direct analogy with $(\mathrm{I} ; 2.15)$.

All those results in Sections 2 and 3 of Part I which do not depend on the calculated values of $c_{2 r}(\alpha)$ hold for this region of integration if we replace the terms as indicated:

$$
\begin{aligned}
a^{2 r} c_{2 r}(R) & \rightarrow d_{2 r}(R), \\
a & \rightarrow \frac{1}{2} .
\end{aligned}
$$

In particular, the error coefficients of a composite rule,

$$
R^{(1)}=\sum \xi_{i} R\left(\alpha_{i}\right),
$$

are given by

$$
d_{2 r}\left(R^{(1)}\right)=\sum \xi_{i} d_{2 r}\left(\alpha_{i}\right),
$$

and the error expansion takes the form

$$
R^{(1)} f-I^{(1)} f=d_{2}\left(R^{(1)}\right) I^{(1)} f^{(2)}+d_{4}\left(R^{(1)}\right) I^{(1)} f^{(4)}+\cdots .
$$

In Section 3 of Part I we treat the error coefficients of $n$-dimensional rules. We may derive the error expansion for an $n$-dimensional composite rule,

$$
R^{(n)}=\sum \xi_{i} \mathcal{Q}_{i}{ }^{(n)},
$$

where

$$
\mathbb{R}_{i}^{(n)}=\mathcal{R}\left(\alpha_{i 1}, \alpha_{i 2}, \cdots, \alpha_{i n}\right),
$$


in terms of the error coefficients $d_{2 \varepsilon}\left(\alpha_{i j}\right)$. The result is

$$
R^{(n)} f-I^{(n)} f=\sum_{s=1}^{\infty} \sum_{\Sigma_{i}==_{s}} d_{2 s_{1} 2 \delta_{2} \cdots 2 \delta_{n}}\left(R^{(n)}\right) I^{(n)} \frac{\partial^{28} f}{\partial^{28_{1}} x_{1} \partial^{2 s_{2}} x_{2} \cdots \partial^{2 s_{n}} x_{n}}
$$

where

$$
d_{2 s_{1} 2 s_{2} \cdots 2 s_{n}}\left(R^{(n)}\right)=\sum \xi_{2} d_{2 s_{1} 2 s_{2} \cdots 2 s_{n}}\left(\mathcal{R}_{i}{ }^{(n)}\right)
$$

and

$$
d_{2 s_{1} 2 s_{2} \cdots 2 s_{n}}\left(\mathscr{R}_{i}^{(n)}\right)=\frac{1}{n !} \sum_{P} d_{2 s_{1}}\left(\alpha_{i P_{1}}\right) d_{2 s_{2}}\left(\alpha_{i P_{2}}\right) \cdots d_{2 s_{n}}\left(\alpha_{i P_{n}}\right) .
$$

Here the integers $P_{1}, P_{2}, \cdots, P_{n}$ are a permutation of the numbers $1,2, \cdots, n$, and the summation symbol indicates that we sum over all such permutations. We may also derive the formula for the error coefficient of the convolution product of one-dimensional composite rules (given by I; 3.25 ), which includes the important special case

$$
d_{2 s_{1} 2 s_{2} \cdots 2 s_{n}}\left(\left(R^{(1)}\right)^{n}\right)=d_{2 s_{1}}\left(R^{(1)}\right) d_{2 s_{2}}\left(R^{(1)}\right) \cdots d_{2 s_{n}}\left(R^{(1)}\right) .
$$

4. Applications of Rule Extension. In Section 4 of Part II we introduced a set notation which helped to clarify the situation regarding the degrees of an $s$-dimensional rule $R^{(s)}$ and an $r$-dimensional rule $R^{(t)}$ in the case in which

$$
R^{(r)} \Rightarrow R^{(s)} \text {. }
$$

The principal result of that section is:

Theonem 4.2. If $R^{(r)} \Rightarrow R^{(s)}$ and $R^{(s)}$ is of degree $2 t+1$, the degree of $R^{(r)}$ is at least $2 t^{\prime}+1$, where $t^{\prime}=\min (s, t)$.

The proof of this theorem rests principally on the results about rule projection and rule extension given in Section 2 and 3 of Part II. The dependence on the integration operator is through equation (II ; 4.2) which states

$$
I^{(r)} f\left(x_{1}, x_{2}, \cdots, x_{s}\right)=I^{(s)} f\left(x_{1}, x_{2}, \cdots x_{s}\right) .
$$

This equation is also true if $I^{(r)}$ is defined as in Section 2 of this paper. As the theory of rule projection and rule extension is independent of the region of integration, it follows that all the results of Section 4 of Part II apply also for this integral, and, in particular, Theorem 4.2 is true.

The systematic construction of high-dimensional integration rules may be effected by following the same procedure as described in Sections 5 to 7 of Part II. In the examples, the role of the Gauss-Legendre quadrature formula is taken over by the Gauss-Hermite quadrature formula. This is of exactly the same form, being a $(t+1)$-point rule of degree $2 t+1$, namely,

$$
G_{t+1}=\sum_{i=1}^{[(t+1) / 2]} \zeta_{i} R\left(\beta_{i}\right) .
$$

The values of $\zeta_{i}$ and $\beta_{i}$ are available in the literature. (See, for example, Kopal [1].)

The rules $E_{t}{ }^{n}(0)\left(G_{t+1}\right)^{t}$ are as before $n$-dimensional integration rules of degree $2 t+1$. The only difference is that different values of $\zeta_{i}$ and $\beta_{i}$ are used. In the 
example in Section 6 of Part II, where we determine $E_{2}{ }^{n}(0)\left(G_{3}\right)^{2}$, we have to replace $(\mathrm{II} ; 6.6)$ and $(\mathrm{II} ; 6.7)$ by

$$
\begin{aligned}
G_{3} & =\frac{2}{3} R(0)+\frac{1}{3} R(\sqrt{ } 6), \\
\left(G_{3}\right)^{2} & =\frac{4}{9} R(0,0)+\frac{4}{9} R(0, \sqrt{ } 6)+\frac{1}{9} R(\sqrt{ } 6, \sqrt{ } 6) .
\end{aligned}
$$

The expression for $E_{2}^{\prime n}(0)\left(G_{3}\right)^{2}$ is the same as in $(I I ; 6.8)$ and this leads to the rule

$$
\begin{aligned}
& E_{2}{ }^{n}(0)\left(G_{3}\right)^{2}=\frac{1}{18}\left[\left(n^{2}-7 n+18\right)\{R(0)\}^{n}\right. \\
& \left.\quad+2 n(4-n)\{\Re(0)\}^{n-1} * \mathbb{R}(\sqrt{ } 6)+n(n-1)\{\Re(0)\}^{n-2} * \Re(\sqrt{ } 6, \sqrt{ } 6)\right],
\end{aligned}
$$

in direct analogy to (II; 6.9 ).

The rules $\tilde{G}_{t+1}^{(n)}$ may be obtained in exactly the same way as in Section 7 of Part II. Here we have to replace $(I I ; 7.8)$ by

$$
I^{(t)} x_{1}^{2} x_{2}^{2} \cdots x_{t}^{2}=\left(\frac{1}{2}\right)^{t} .
$$

Thus $\phi$ in $(I I ; 7.9)$ is given by

$$
\phi=1 /\left(2{\beta_{1}}^{2}\right)^{t} .
$$

With this adjustment the subsequent formulas and rules are valid. We find

$$
\bar{G}_{t+1}^{(n)}=E_{t-1}^{n}(0)\left(G_{t+1}\right)^{t-1}+\frac{1}{\left(2 \beta_{1}\right)^{t}}\left[E_{t}{ }^{n}(0)\left\{R\left(\beta_{1}\right)\right\}^{t}-E_{t-1}^{n}(0)\left\{R\left(\beta_{1}\right)\right\}^{t-1}\right]
$$

and

$$
\begin{gathered}
\nu\left(\bar{G}_{t+1}^{(n)}\right) E=1+t\left(\begin{array}{l}
n \\
1
\end{array}\right)+\cdots+t^{t-1}\left(\begin{array}{c}
n \\
t-1
\end{array}\right)+2^{t}\left(\begin{array}{l}
n \\
t
\end{array}\right) \quad(t \text { even }), \\
=1+(t+1)\left(\begin{array}{l}
n \\
1
\end{array}\right)+\cdots+(t+1)^{t-1}\left(\begin{array}{c}
n \\
t-1
\end{array}\right) \\
+2^{t}\left(\begin{array}{l}
n \\
t
\end{array}\right) \quad(t \text { odd }) .
\end{gathered}
$$

In the example $\bar{G}_{4}{ }^{(n)}$ given in (II ; 7.20) the incidental parameters are different, though the rule is of identical form. These parameters are:

$$
\begin{aligned}
\zeta_{1} & =(3-\sqrt{ } 6) / 6, \\
\zeta_{2} & =(3+\sqrt{ } 6) / 6, \\
{\beta_{1}}^{2} & =2(3+\sqrt{ } 6), \\
{\beta_{2}}^{2} & =2(3-\sqrt{ } 6), \\
\phi & =1 / 8 \beta_{1}{ }^{6} .
\end{aligned}
$$

The number of points required by any of these rules is, in general, the same as that stated for the corresponding rule in Part II; exceptionally, the vanishing of a coefficient of a basic rule leads to a reduction. An example of this occurs in (4.6) above. There,

$$
\begin{aligned}
\nu\left({E_{2}}^{n}(0)\left(G_{3}\right)^{2}\right) & =2 n^{2}+1, \quad n=2,3,5,6, \cdots, \\
& =25, \quad n=4,
\end{aligned}
$$


the exceptional case $n=4$ occurring as a result of the factor $(n-4)$ in the coeffcient of one of the basic rules.

The rules $\bar{G}_{t}^{(n)}$ establish for this region of integration a conjecture (Thacher [5]). This conjecture may be stated in exactly the same form as in Section 8 of Part II; there exists a set of integration rules $\bar{G}_{t+1}^{(t)}, \bar{G}_{t+1}^{(t+1)}, \cdots$ in $t, t+1, \cdots$ dimensions with the property

$$
\operatorname{Lim}_{n \rightarrow \infty} \nu\left(\bar{G}_{t+1}^{(n)}\right) / 2^{t}\left(\begin{array}{l}
n \\
t
\end{array}\right)=1 .
$$

5. Discussion. The integration rules derived above are all of the type defined in Section 1 of Part I; that is, they are all,invariant under interchange of coordinates and the alteration of the sign of any coordinate. We refer to this type of rule as one having hypercubic symmetry.

The space considered here is spherically symmetric. No advantage of this full symmetry has been taken in constructing these rules. Rather, we have used only the (much weaker) hypercubic symmetry. One might naturally expect such rules to be inferior or less economic in terms of function evaluations than rules derived on the basis of full spherical symmetry. Stroud and Secrest [4] have published rules for values of $n$ and $t$ both less than 4 which do not have hypercubic symmetry and which use fewer points than any corresponding rules which do have hypercubic symmetry. However, in the case of $n \geqq t \geqq 4$, the presently available rules derived using the spherical symmetry are of a product type and require $\nu\left(S_{P}\right)$ points, where

$$
\begin{aligned}
\nu\left(S_{P}\right) & =(t+1)^{n} \quad(t \text { odd }), \\
& =(t+1)^{n}-t^{n}+1 \quad(t \text { even }) .
\end{aligned}
$$

(See Stroud and Secrest [4], Section 3.) This is marginally fewer than the number required by the product Gaussian:

$$
\nu\left(\left(G_{t+1}\right)^{n}\right)=(t+1)^{n} .
$$

However, the rules derived in the present paper, which have only hypercubic symmetry, require only

$$
\nu\left(\bar{G}_{t+1}^{(n)}\right) \sim 2^{t}\left(\begin{array}{l}
n \\
t
\end{array}\right)
$$

(See Eq. (4.10).)

Thus the present situation is that the most economical rules available for this spherically symmetric region have only hypercubic symmetry when $n \geqq t \geqq 4$.

Applied Mathematics Department

University of New South Wales

Kensington, N.S.W., Australia

1. Z. Kopal, Numerical Analysis, Wiley, New York and Chapman and Hall, London, 1955. MR 17, 1007.

2. J. N. LYNESS, "Symmetric integration rules for hypercubes. I. Error coefficients," Math. Comp., v. 19, 1965, pp. 260-276.

3. J. N. LyNESS, "Symmetric integration rules for hypercubes. II. Rule projection and rule extension," Math. Comp., v. 19, 1965, pp. 394-407.

4. A. H. STROUD \& D. SECREST, "Approximate integration formulas for certain spherically symmetric regions," Math. Comp., v. 17, 1963, pp. 105-135.

5. H. C. Thacher, JR., "Optimum quadrature formulas in $s$ dimensions," MTAC, v. 11, 1957, pp. 189-194. 